\title{
An Optimization Model for the Management of Security Risks in Banking Companies
}

by

Ulrich Faisst, Oliver Prokein ${ }^{1}$

April 2005

presented at: Müller, G., Lin, K.-J., Proceedings of the 7th IEEE International Conference on E-Commmerce Technology (CEC) 2005, München, July 2005, IEEE Computer Society Press, Los Alamitos, CA, 2005, p.266-273

\footnotetext{
${ }^{1}$ Institut für Informatik und Gesellschaft, Universität Freiburg
} 


\section{An Optimization Model for the Management of Security Risks in Banking Companies}

\author{
Ulrich Faisst \\ Department of Information Systems \& \\ Financial Engineering, \\ Business School \\ University of Augsburg \\ email: Ulrich.Faisst@,wiwi.uni-augsburg.de
}

\author{
Oliver Prokein \\ Institute of Computer Science and \\ Social Studies, \\ Department of Telematics \\ University of Freiburg \\ email: oliver.prokein@iig.uni-freiburg.de
}

\begin{abstract}
Increasing importance of information and communication technologies (ICT), new regulatory obligations (e.g. Basel II) and growing external risks (e.g. hacker attacks) put Security Risks in the management focus of banking companies. The management has to decide whether to accept Expected Losses or to invest into Technical Security Mechanisms in order to decrease the frequency of events or to invest in Insurance Policies in order to lower the severity of events. This paper contributes to the development of an optimization model that aims to determine the optimal amount to be invested in technical Security Mechanisms and Insurance Policies. Furthermore the model considers budget and risk limits as constraints and is supposed to help practitioners in controlling Security Risks.
\end{abstract}

\section{Introduction}

Due to the increasing virtualization of business processes and the cumulative adoption of ICT involved, Security Risks have lately gained in significance. The "Electronic Commerce Enquête IV" inquiry carried out in August 2004 concluded that the majority of German banking companies plan to increase the investments in ICT within the next two years [12].

However, the rising deployment of ICT implicates increasing Security Risks. Increasing Investments in Technical Security Mechanisms and Insurance Policies generally lead to lower Expected Losses and Opportunity Costs of the Regulatory Capital Charge, et vice versa [4]. Thus, a trade-off exists between the Expected Losses and the Opportunity Costs of the Regulatory Capital Charge on the one hand and the
Investments in Technical Security Mechanisms and Insurance Policies on the other.

In practice, such investment decisions depend on explicit responsibilities within a company. In case, where an explicit responsibility exists, the decisionmaker will tend to make every possible investment within his budget, although holistically viewed not every investment is profitable. If no explicit responsibility exists, the decision-maker will tend to minimize costs and therefore neglects further investments, although such investments are profitable in a holistic view.

This paper aims at developing an optimization model that is able to map the described trade-off between the Expected Losses and the Opportunity Costs of the Regulatory Capital Charge on the one hand and the Investments in Security Mechanisms and Insurance Policies on the other in a decision calculation. Moreover, the model helps to allocate available budgets to Security Mechanisms (ex-ante prevention) and into Insurance Policies (ex-post risk transfer) in an efficient way. In order to lay the basic principles for the model we will first portray the risk management process.

\section{The Risk Management Process}

The activities of risk management can be illustrated according to the risk management process. The process contains the four phases of identification, quantification, controlling and monitoring [10]. The risk management process is illustrative and may not be interpreted as a unique operational sequence. In practice, it is necessary to improve the process continuously and challenge the results critically. 


\section{Identification Phase}

Within the scope of the identification phase, the Security Risks are identified and classified. Security in ICT covers the wide range from the physical protection of the hardware to the protection of personal data against deliberate attacks [8]. In an open information system like the Internet, one cannot assume, that all parties involved (such as communication partners, services providers etc.) trust or even know each other [9]. Therefore, the analysis of security requires not only the observation of external attackers but also the inclusion of all parties involved as potential attackers. The concept of multilateral security [9] considers the security requirements of all parties involved. The Security Risks result from the threat of the so-called four protection goals of multilateral security [11]:

- Loss of Confidentiality, i.e. the risk of unauthorized gain of information.

- Loss of Integrity, i.e. the risk of unauthorized modification or erasure of information and data.

- Loss of Accountability, i.e. the risk of illegal irresponsibility.

- Loss of Availability, i.e. the risk of unauthorized impairment of the functionality.

Table 1: Economic impacts of attacks

\begin{tabular}{|l|l|l|}
\hline $\begin{array}{c}\text { Protection } \\
\text { Goals }\end{array}$ & Selected Attacks & \multicolumn{1}{|c|}{$\begin{array}{c}\text { Potential } \\
\text { Economic } \\
\text { Impacts }\end{array}$} \\
\hline $\begin{array}{l}\text { Confiden- } \\
\text { tiality }\end{array}$ & $\begin{array}{l}\text { Hacker-Attacks, } \\
\text { Industry-Spying, } \\
\text { Access Misuse etc. }\end{array}$ & $\begin{array}{l}\text { Loss of Competitive } \\
\text { Advantage, Liability } \\
\text { Claims of Third, } \\
\text { Punishments etc. }\end{array}$ \\
\hline Integrity & $\begin{array}{l}\text { Sabotage, Man-in-the- } \\
\text { Middle-Attack, } \\
\text { Computer Bug etc. }\end{array}$ & $\begin{array}{l}\text { Loss of Data, Business } \\
\text { Interruption, Sales } \\
\text { Shortfall etc. Imager Image, }\end{array}$ \\
\hline $\begin{array}{l}\text { Account- } \\
\text { ability }\end{array}$ & $\begin{array}{l}\text { IP-Spoofing, Social } \\
\text { Hacking, Inadequate } \\
\text { Access Control etc. }\end{array}$ & $\begin{array}{l}\text { Lusiness Interruption, } \\
\text { Liability Claims of } \\
\text { Third etc. }\end{array}$ \\
\hline Availability & $\begin{array}{l}\text { DDOS, Virus, Hard } \\
\text { Failure etc. }\end{array}$ & $\begin{array}{l}\text { Loss of Recovery, } \\
\text { Loss of Market Share } \\
\text { etc. }\end{array}$ \\
\hline
\end{tabular}

Table 1 illustrates selected attacks and their potential economic impacts. The probability of loss occurrence arises from the observed attacks, the amount of losses from the economic impacts.

\section{Quantification Phase}

The identified Security Risks are measured by the use of different methods within the quantification phase [3]. So far, no quantification model has been developed for the measurement of the Security Risks, defined above. These Security Risks are however a subset of Operational Risks. The Basel Committee on
Banking Supervision defines Operational Risk as the risk of loss resulting from inadequate or failed internal processes, people and systems or from external events [2]. ${ }^{1}$ For the measurement of this Operational Risk the committee suggests five different quantification methods in order to determine the Regulatory Capital Charge. The models reach from simple, factor-based approaches to complex stochastic loss distribution models based on the Value-at-Risk [1], [2]. Beyond that, further methods exist for the quantification of Operational Risks, as for instance questioning techniques or causal methods, like Bayesian Belief Networks [8].

\section{Controlling Phase}

Based on the identified and quantified Security Risks, decisions on carrying, decreasing, avoidance as well as the transfer of the Security Risks are made within the controlling phase.

Technical Security Mechanisms can be used to control ex-ante Security Risks. Table 2 illustrates selected Security Mechanisms. We assume that Security Mechanisms are appropriate for reducing the Expected Loss Frequency of successful attacks exante.

Table 2: Protection goals and Security Mechanisms

\begin{tabular}{|l|l|}
\hline \multicolumn{1}{|c|}{$\begin{array}{c}\text { Protection } \\
\text { Goals }\end{array}$} & \multicolumn{1}{|c|}{ Selected Security Mechanisms } \\
\hline Confidentiality & $\begin{array}{l}\text { Symmetric and Asymmetric Cryptography, } \\
\text { Firewalls, VPN, Stenography, Broadcast } \\
\text { etc. }\end{array}$ \\
\hline Integrity & $\begin{array}{l}\text { Digital Signatures, Message Authentication } \\
\text { Codes, Virus Scanner etc. }\end{array}$ \\
\hline Accountability & $\begin{array}{l}\text { Digital Signatures, Public-Key- } \\
\text { Infrastructures, Watermaking etc. }\end{array}$ \\
\hline Availability & $\begin{array}{l}\text { Patches, Backup-Systems, IDS, Physical } \\
\text { Protection, etc. }\end{array}$ \\
\hline
\end{tabular}

Moreover, banking companies are able to transfer the amount of losses ex-post by Insurance Policies. However, due to the characteristics, not every security risk is insurable. Various catalogues of criteria have been developed in the past in order to examine insurability. The five criteria of KARTEN are referred to below [7]:

- The criterion of fortuitousness demands that the event causing the case has to be uncertain and unaffected.

\footnotetext{
This definition includes legal risk, but excludes strategic and reputational risk.
} 
- Unambiguousness assumes that the occurrence and the amount of losses are verifiable in an objective way.

- Estimability targets the problem of insufficient knowledge. An insurance company must be able to estimate the probability of occurrence and the average amount of losses.

- Independence refers to positively related risks that should be excluded so as to ensure a process of fortuity of the insured loss given events of the business in force.

- The last criteria, size refers to the maximum damage that can result from a single risk. Because it is difficult to quantify the damages exactly, insurance companies only agree to cover a certain percentage of the amount of losses.

With regard to these criteria, table 3 illustrates that only threats of the protection goals integrity and availability are insurable [6].

Table 3: Insurability of Security Risks

\begin{tabular}{|l|l|l|}
\hline $\begin{array}{l}\text { Protection } \\
\text { Goals }\end{array}$ & Insurability & Problems \\
\hline Confidentiality & Not Applicable & $\begin{array}{l}\text { Definite Causal Connection, } \\
\text { provability, quantification }\end{array}$ \\
\hline Integrity & Yes & $\begin{array}{l}\text { Low Limits of Coverage, } \\
\text { Expensive Technical Security } \\
\text { Precautions }\end{array}$ \\
\hline Accountability & Not Applicable & $\begin{array}{l}\text { Definite Causal Connection, } \\
\text { Provability }\end{array}$ \\
\hline Availability & Yes & $\begin{array}{l}\text { Low Limits of Coverage, } \\
\text { Expensive } \\
\text { Precautions }\end{array}$ \\
\hline
\end{tabular}

\section{Monitoring Phase}

The monitoring phase encompasses all procedures and techniques, which are necessary for a continuous monitoring of the Security Risks. Thereby it is analyzed, if

- $\quad$ all the occurred events have been prior identified as possible events,

- the distribution of probabilities of occurrence of events and the distribution of severities of losses have been anticipated within the quantification phase,

- the selected controlling measures have lead to the desired results.

This paper focuses on the controlling phase. In the following it will be investigated, which combinations of ex-ante and ex-post controlling measures lead to an efficient solution.

\section{A Controlling Model for Security Risks}

The model aims to solve the trade-off between the Expected Losses and the Opportunity Costs of the Regulatory Capital Charge on the one hand and the Investments in Security Mechanisms and Insurance Policies on the other. Thereby, the amount to be invested in Security Mechanisms and Insurance Policies will be optimized.

\subsection{Assumptions (in italics)}

The time horizon accounts for a single period.

\section{Assumption 1: Independence of a Single Information System}

In the following, an open single information system is regarded. It is assumed that no dependencies exist to other information systems.

\section{Assumption 2: Relevant Cashflow Parameters}

The Expected Total Negative Cashflow $\mu$ of the information system is composed of the items Expected Losses due to Security Risks E(L), the Opportunity Costs of the Regulatory Capital Charge RCC, the Investments in Security Mechanisms $I_{S M}$ and the Investments in Insurance Policies $I_{\text {Ins }}$.

$$
\mu=E(L)+R C C+I_{S M}+I_{\text {Ins }}(1)
$$

The stochastic cashflow item $L$ as well as the deterministic cashflow items $R C C, I_{S M}$ and $I_{\text {Ins }}$ are estimated ex-ante.

\section{Assumption 2a: Expected Losses due to Security Risks}

As mentioned in Chapter 2, the Basel Committee on Banking Supervision proposes five different approaches to quantify Operational Risks, such as Security Risks. The proposed Internal Measurement Approach [2] is modified to quantify the Expected Losses $E(L)$.

$$
\begin{aligned}
& E(L)=E[(a \cdot N) \cdot(b \cdot L G E)] \\
& =(a \cdot E(N)) \cdot(b \cdot L G E) \\
& =(a \cdot \lambda) \cdot(b \cdot L G E)
\end{aligned}
$$



whereas: $E(N)=\lambda:=$ Expected Frequency of Occurrence ${ }^{2}$,
$a:=$ Percentage of Successful Attacks, $L G E:=$ Expected Loss Given Events, $b:=$ Percentage of not Insured Loss Given Events.

According to the Internal Measurement Approach $E(L)$ arises as a result of multiplying the expected frequency of occurrence $\lambda$ by the Expected Loss Given Events LGE (with $L G E>0$ ). For simplicity, we assume constant LGE. We assume, that Investments in Security Mechanisms can reduce the Expected Frequency of Occurrence $\lambda$ ex-ante by the so called Security Level $(S L=1-a)$. The Security Level represents the percentage of prevented attacks through the implementation of Technical Security Mechanisms. In order to make allowance for the impacts of these mechanisms, the Expected Frequency of Occurrence is multiplied by the factor $a$ (whereby $0<a \leq 1$ ) that represents the Percentage of Successful Attacks. We further assume that Investments in Insurance Policies can reduce the amount of losses $L G E$ by the so called represents the percentage of the transferred respective insured Loss Given Events. In order to make allowance for the impacts of Insurance Policies, the Expected Loss Given Events are multiplied by the factor $b$ (whereby $0<b \leq 1$ ) that represents the percentage of not insured loss given events.

The Internal Measurement Approach assumes a binomial-distribution for $\lambda$. However, the binomial distribution approaches the poisson distribution for large numbers of observed attacks with a small probability of occurrence. The poisson distribution exhibits the characteristic that the variance corresponds to the expectancy value.

$$
\sigma^{2}=a \cdot \lambda(3)
$$

For constant LGE, the standard deviation of Security Risks is given by:

$$
\sigma_{S R}=\sqrt{a \cdot \lambda}(b \cdot L G E)
$$
Insurance Level $(I L=1-b)$. The Insurance Level

\section{Assumption 2b: Opportunity Costs of Regulatory Capital Allocation}

According to the Internal Measurement Approach [1], the Regulatory Capital Charge $K$ is given by:

$$
K=\gamma \cdot E(L)(5)
$$

The Capital Charge $K$ arises as a result of multiplying $E(L)$ by the so-called gamma-factor $\gamma^{3}$. With an interest rate of $r$, the Opportunity Costs of the Regulatory Capital Charge RCC are given by:

$$
R C C=r \cdot K=r \cdot \gamma \cdot E(L)(6) .
$$

The Opportunity Costs $R C C$ exhibit a deterministic character. The standard deviation $\sigma_{R C C}$ is therefore given by:

$$
\sigma_{R C C}=0(7)
$$

\section{Assumption 2c: Investments in Security Mechanisms}

We assume that the Probability of Loss Occurring $\lambda$ can be reduced ex-ante by implementing Security Mechanisms.

$$
I_{S M}=\left(\frac{\lambda \cdot L G E}{[a \cdot \lambda \cdot L G E]^{\beta}}-1\right)
$$

$$
\begin{array}{ll}
\text { whereby } & I_{S M}=0 \text { for } a=\beta=1 \\
\text { and } & I_{S M}>0 \text { for } 0<a<1 \wedge 0<\beta<1 .
\end{array}
$$

According to assumption $2 \mathrm{a}$ and equation (8), there is an inversely proportional relationship between the Investments in Security Mechanisms $I_{S M}$ and the Percentage of Successful Attacks $a$ for a constant calibration factor $\beta$. This calibration factor determines the sensitivity of the relationship (whereby $0<\beta \leq 1$ ). According to assumption $2 \mathrm{a}$, increasing Investments in Security Mechanisms $I_{S M}$ implicate decreasing Expected Losses $E(L)$ et vice versa.

Similar to the Opportunity Costs, the Investments in Security Mechanisms $I_{S M}$ exhibit a deterministic character. The standard deviation is therefore given by:

$$
\sigma_{S M}=0(9)
$$

\footnotetext{
2 In the following, we refer to $\mathrm{E}(\mathrm{N})$ as $\lambda$. The Basel Committee on Banking Supervision defines the Expected Frequency of Occurrence $E(N)=\lambda$ as a the product of an Exposure Indicator (EI) with the Probability of Events (PE) per exposure. Therefore $N$ is a random variable of the Loss Frequency.
}

3 The gamma-factor translates the estimate of Expected Losses into a
Capital Charge [1]. 


\section{Assumption 2d: Investments in Insurance Policies}

If the Security Risks fulfill the criteria of insurability, banking companies are able to reduce the extent of damage ex-post by Investments in Insurance Policies.

$$
I_{\text {Ins }}=\left(\frac{\lambda \cdot L G E}{[b \cdot \lambda \cdot L G E]^{\delta}}-1\right)(10)
$$

whereby

$$
\begin{aligned}
& I_{\text {Ins }}=0 \text { for } b=\delta=1 \\
& I_{\text {Ins }}>0 \text { for } 0<b<1 \wedge 0<\delta<1 .
\end{aligned}
$$

and

Analogous to the Investments in Security Mechanisms we assume an inversely proportional relationship between the Investments in Insurance Policies $I_{\text {Ins }}$ and the percentage of not insured Loss Given Events $b$ for a constant calibration factor $\delta$. This calibration factor determines the sensitivity of the relationship (whereby $0<\delta \leq 1$ ). According to assumption $2 \mathrm{a}$, increasing Investments in Insurance Policies $I_{\text {Ins }}$ implicate decreasing $E(L)$, et vice versa.

The Investments in Insurance Policies $I^{S M}$ exhibit a deterministic character and the standard deviation is given by:

$$
\sigma_{\text {Ins }}=0(11) \text {. }
$$

\section{Assumption 3: Solution Space with continuous $\sigma$ and its transformation on $\mu(\sigma)$ :}

We assume that any number of $\sigma \in(0, \infty)$ exists and the corresponding cashflows can be mapped through the continuous function $\mu(\sigma) .{ }^{4}$ Only one $\sigma$ can be realized, combinations are not possible.

\subsection{Determining the Optimal Security and Insurance Level}

In order to determine the optimal Security- and Insurance Level $\left(S L^{*}, I L^{*}\right)$ and the corresponding optimal amount to be invested in technical Security Mechanisms $I_{S M}^{*}$ and Insurance Policies $I_{\text {Ins }}^{*}$, we assume a risk neutral decision-maker that aims at minimizing his Expected Total Negative Cashflow $\mu$.

The Expected Total Negative Cashflow is obtained by the substitution of (2), (6), (8) and (10) in (1):

4 Thus, it is assumed that any number of $\sigma$ can be obtained. In reality only a finite number of discrete values of $\sigma$ exist. Another simplification is the assumption that for any number of $\sigma$ a continuous function $\mu(\sigma)$ exists.

$$
\begin{aligned}
& \mu=(1+r \cdot \gamma)(a \cdot \lambda)(b \cdot L G E) \\
& +\frac{\lambda \cdot L G E}{(a \cdot \lambda \cdot L G E)^{\beta}}+\frac{\lambda \cdot L G E}{(b \cdot \lambda \cdot L G E)^{\delta}}-2
\end{aligned}
$$

The derivation of equation (12) with respect to $a$ is given by:

$$
\begin{gathered}
\frac{\partial \mu}{\partial a}=\lambda(1+r \gamma)(b L G E)-\beta \frac{\lambda \cdot L G E}{(\lambda \cdot L G E)^{\beta} \cdot a^{\beta+1}}(13), \\
\frac{\partial^{2} \mu}{\partial^{2} a}=\beta(\beta+1) \frac{\lambda \cdot L G E}{(\lambda \cdot L G E)^{\beta} \cdot a^{\beta+2}}>0
\end{gathered}
$$

Equation (13) fulfills the necessary and (14) the sufficient condition of a minimum of the Expected Total Negative Cashflow. Transformation of (13) leads to:

$$
a=\frac{\left(\frac{\beta \cdot \lambda \cdot L G E}{b \cdot(1+r \gamma)}\right)^{\frac{1}{\beta+1}}}{\lambda \cdot L G E}(15) .
$$

The derivation of the variable $b$ is obtained analogous and is given by:

$$
b=\frac{\left(\frac{\delta \cdot \lambda \cdot L G E}{a \cdot(1+r \gamma) \cdot}\right)^{\frac{1}{\delta+1}}}{\lambda \cdot L G E}(16) .
$$

Substitution of (16) in (15) and (15) in (16) leads to

$$
\begin{aligned}
& a^{*}=(\lambda \cdot L G E)^{\frac{\delta-\beta(\delta+1)}{\beta(\delta+1)+\delta}} \cdot\left(\frac{\beta^{(1+\delta)}}{\delta \cdot(r \cdot \gamma+1)^{\delta}}\right)^{\frac{1}{\beta \cdot(\delta+1)+\delta}} \\
& b^{*}=(\lambda \cdot L G E)^{\frac{\beta-\delta(\beta+1)}{\beta \cdot(\delta+1)+\delta}} \cdot\left(\frac{\delta^{(1+\beta)}}{\beta \cdot(r \cdot \gamma+1)^{\beta}}\right)^{\frac{1}{\beta(\delta+1)+\delta}}
\end{aligned}
$$

The optimal Security Level $S L^{*}$ and Insurance Level $I L^{*}$ are (see assumption 2 a)

$$
\begin{aligned}
S L^{*} & =1-a^{*}(19), \\
I L^{*} & =1-b^{*}(20),
\end{aligned}
$$

and therefore obtained by substitution of (17) in (19) and (18) in (20): 


$$
\begin{aligned}
& S L^{*}=1-\left[(\lambda \cdot L G E)^{\frac{\delta-\beta(\delta+1)}{\beta(\delta+1)+\delta}} \cdot\left(\frac{\beta^{(1+\delta)}}{\delta \cdot(r \cdot \gamma+1)^{\delta}}\right)^{\frac{1}{\beta \cdot(\delta+1)+\delta}}\right] \\
& I L^{*}=1-\left[(\lambda \cdot L G E)^{\frac{\beta-\delta(\beta+1)}{\beta \cdot(\delta+1)+\delta}} \cdot\left(\frac{\delta^{(1+\beta)}}{\beta \cdot(r \cdot \gamma+1)^{\beta}}\right)^{\frac{1}{\beta(\delta+1)+\delta}}\right]
\end{aligned}
$$

The minimum of the Expected Total Negative Cashflow $\mu^{*}\left(a^{*}, b^{*}\right)$ is obtained by the substitution of the equations (17) and (18) in equation (12). In doing so, it is further possible to determine the optimal amount to be invested in Security Mechanisms $I_{S M}^{*}$ and Insurance Policies $I_{\text {Ins }}^{*}$.

$$
\begin{aligned}
& I_{S M}^{*}=\frac{\lambda \cdot L G E}{\left[(\lambda \cdot L G E)\left(\frac{2 \delta}{(1+\delta) \beta+\delta}\right) \cdot\left(\frac{\beta^{(1+\delta)}}{\delta \cdot(1+r \cdot \gamma)^{\delta}}\right)\left(\frac{1}{(1+\delta) \beta+\delta}\right)\right]^{\beta}}-1 \\
& I_{\text {Ins }}^{*}=\frac{\lambda \cdot L G E}{\left[(\lambda \cdot L G E)\left(\frac{2 \beta}{(1+\delta) \beta+\delta}\right) \cdot\left(\frac{\delta^{(1+\beta)}}{\beta \cdot(1+r \cdot \gamma)^{\delta}}\right)\left(\frac{1}{(1+\delta) \beta+\delta}\right)\right]^{\delta}}-1
\end{aligned}
$$

The mapped area in figure 1 illustrates all possible $\mu(a, b)$-combinations and the corresponding security and Insurance Level ( $S L, I L)$ for given values of $\beta$ and $\delta$. However, there is only one minimum in $\mu^{*}\left(a^{*}, b^{*}\right)$ and therefore only one efficient $\left(S L^{*}, I L^{*}\right)$ solution.

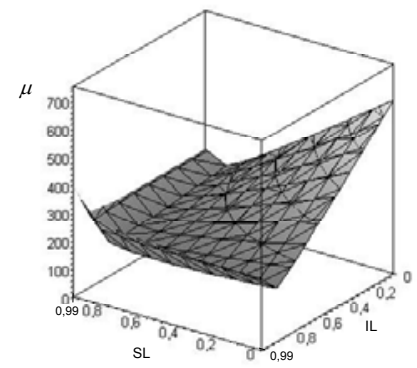

Figure 1: Minimum of the Expected Total Negative Cashflow. ${ }^{5}$

\footnotetext{
5 According to assumption $2 \mathrm{a}$, the domains of $\mathrm{a}, \mathrm{b}$ and $\mathrm{SL}$, IL respectively are given by $a, b \in] 0,1]$ and $S L, I L \in[0,1[$. The closer $S L, I L$ approaches to 1 , the greater the Expected Total
}

Example 1: Consider the following instance for the model: $\beta=0,2, \quad \delta=0,6, \quad r=0,1, \quad L G E=1.000, \quad \lambda=0,3 \quad$ and $\gamma=7,3$.

The optimal Security and Insurance Level are given by $S L^{*}=0,58$ (with $a^{*}=0,42$ ) and $I L^{*}=0,9$ (with $b^{*}=0,1$ ). Therefore a banking company would invest $I_{S M}^{*}=112,99$ in technical Security Mechanisms and $I_{I n s}^{*}=36,99$ in Insurance Policies. The Expected Loss equals the value $E(L)=13,18$ and the Opportunity Costs of the Regulatory Capital Charge $R C C=9,62$. Therefore the minimum of the Expected Total Negative Cashflow is given by $\mu^{*}=172,78$.

Result 1: For any given values of $(\beta, \delta)$ only one minimum of the Expected Total Negative Cashflow $\mu^{*}\left(a^{*}, b^{*}\right)$, respective $\left(S L^{*}, I L^{*}\right)$-solution exists.

\subsection{Constraints and their Impacts}

In practice, constraints like limits for Regulatory Capital Charge and Budget Limits affect the controlling of Security Risks. We will further analyze the impacts of constraints on the minimal Expected Total Negative Cashflow $\mu^{*}$.

In order to analyze the impacts, we will first transform the Expected Total Negative Cashflow in an equation dependent on the standard deviation. The standard deviation of the Expected Total Negative Cashflow $\sigma_{E T N C}$ arises by considering (3), (7), (9) and (11):

$$
\begin{gathered}
\sigma_{E T N C}=\sigma_{S R}=\sqrt{a \cdot \lambda}(b \cdot L G E)(23) . \\
\Rightarrow a \cdot \lambda=\frac{\sigma_{E T N C}^{2}}{(b \cdot L G E)^{2}}
\end{gathered}
$$

In the following, $\sigma_{\text {ETNC }}$ is denoted as $\sigma$.

We obtain $E L, R C C$, and $I^{S M}$ in dependence of $\sigma$ by the substitution of (24) in (2), (6) and (8):

$$
\begin{aligned}
E L & =\frac{\sigma^{2}}{b \cdot L G E}(25) \\
R C C & =r \cdot \gamma \frac{\sigma^{2}}{b \cdot L G E}
\end{aligned}
$$

Negative Cashflow $\mu$. Therefore, $\mu$ is not limited and can rise infinitely. For illustration reasons, the plotted graph shows all the $S L, I L$-combinations within the domain [0, 0.99]. 


$$
I^{S M}=\left(\frac{b}{\sigma}\right)^{2 \beta} \cdot \lambda \cdot L G E^{1+\beta}-1
$$

The Expected Total Negative Cashflow $\mu(\sigma)$ is obtained based on (25), (26), (27) and (10):

$$
\begin{aligned}
& \mu=(1+r \cdot \gamma) \cdot \frac{\sigma^{2}}{b \cdot L G E}+\left(\frac{b}{\sigma}\right)^{2 \beta} \cdot \lambda \cdot L G E^{\beta+1} \\
& +\frac{b \cdot L G E}{(b \cdot \lambda \cdot L G E)^{\delta}}-2
\end{aligned}
$$

Equation (26) describes $\mu(\sigma)$ as a continuous function in dependence of $\sigma . \mu(\sigma)$ thereby maps the domain $\sigma \in(0, \infty)$ well defined on $\mu(\sigma) \in\left(\mu^{*}, \infty\right)$. Figure 2 illustrates the trade-off between the Expected Losses $E(L)$ and the Opportunity Costs of the Regulatory Capital Charge $R C C$ on the one hand and the Investments in Security Mechanisms $I^{S M}$ and Insurance Policies $I^{\text {Ins }}$ on the other. The Expected Total Negative Cashflow $\mu(\sigma)$ thereby possesses only one minimum in $\mu^{*}\left(\sigma^{*}\right)$.

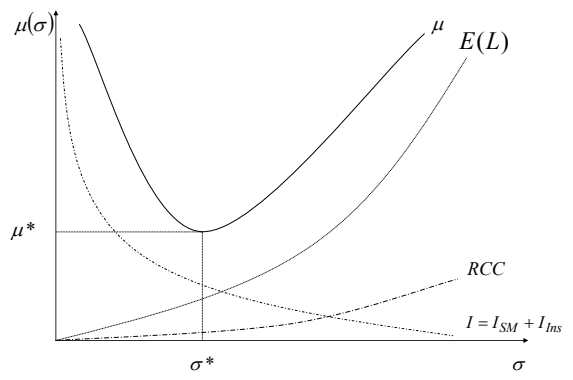

Figure 2: Trade-off between $E(L)$ and $R C C$ on the one hand and $I_{S M}$ and $I_{I n s}$ on the other.

Example 2: Analogous to example 1, we consider the following instance for the model: $\beta=0,2$, $\delta=0,6, \quad r=0,1, \quad L G E=1.000, \quad \lambda=0,3, \quad \gamma=7,3$, $S L^{*}=0,58 \quad$ (with $a^{*}=0,42$ ), $I L^{*}=0,9 \quad$ (with $b^{*}=0,1$ ). and $\mu^{*}=172,78$. Substituting these values in (26) leads to an optimal risk level amounting to $\sigma^{*}=36,17$.

As mentioned above, the minimum of the Expected Total Negative Cashflow $\mu^{*}\left(a^{*}, b^{*}\right)$, respectively $\left(S L^{*}, I L^{*}\right)$-solution, is derived by equation (12) in connection with (19) and (20). The appropriate optimal risk level $\sigma^{*}$ can be determined by equation (26) in conjunction with $\mu^{*}$.

\section{Constraint 1: Limits of the Regulatory Capital Charge}

Composing Limits of the Regulatory Capital Charge determines the amount of risks a banking company is prepared to carry. We now assume that a banking company defines a Limit of the Regulatory Capital Charge LRCC for a single information system. In doing, so the amount of feasible solutions is restricted. In order to illustrate the impacts on the Expected Total Negative Cashflow, we consider two different Limits of the Regulatory Capital Capital $\operatorname{LRCC}_{i}(\mathrm{i}=1,2)$.

The two limits $L R C C_{1,2}$ are represented in figure 2. $L R C C_{1}$ cuts the Expected Total Negative Cashflow in $\left(\mu_{1}, \sigma_{1}\right)$. In this case, the banking company is further able to realize the global minimum of the Expected Total Negative Cashflow in $\left(\mu^{*}, \sigma^{*}\right)$. Therefore, the Limit of the Regulatory Capital Charge $L R C C_{1}$ does not tap the full potential $\left(\sigma^{*}<\sigma_{1}\right)$.

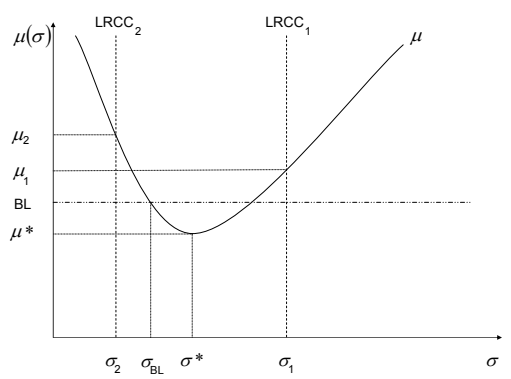

\section{Figure 3: Consideration of Budget Limits and limits of Regulatory Capital Charge.}

However, in the second case the limit $L R C C_{2}$ cuts the Expected Total Negative Cashflow in the suboptimal solution $\left(\mu_{2}, \sigma_{2}\right)$. The Expected Total Negative Cashflow $\mu_{2}$ is greater than $\mu^{*}$, the corresponding risk is accordingly smaller $\left(\sigma_{2}<\sigma^{*}\right)$.

Result 2: In case the $L R C C$ exceeds the optimal solution $\left(\mu^{*}, \sigma^{*}\right)$, a banking company can further realize the minimal Expected Total Negative Cashflow $\left(\mu^{*}, \sigma^{*}\right)$. However, if the $L R C C$ is smaller than $\left(\mu^{*}, \sigma^{*}\right)$ a banking company can only realize suboptimal solutions. 
In addition to the Limit of the Regulatory Capital Charge, Budget Limits are further constraints. Their impacts are analyzed in the following.

\section{Constraint 2: Budget Limits}

Budget Limits serve as a limitation of the payments in business areas or in our case in information systems. Analogous to the Limits of the Regulatory Capital Charge, Budget Limits restrict the amount of feasible solutions. Budgeting generally considers the Expected Losses $E(L)$ ex-ante. Anyhow, SR is a random variable that can exceed ex-post the defined Budget Limit. The amount exceeded can be covered through equity capital. Figure 3 illustrates the impact of the Budget Limit $B L$ exemplarily. $B L$ cuts the Expected Total Negative Cashflow in the suboptimal solution $\left(\mu_{B L}, \sigma_{B L}\right)$. In this case, a banking company can further realize the optimal solution $\left(\mu^{*}, \sigma^{*}\right)$. Assumed that the Budget Limit $B L$ is smaller than the optimal solution $\left(\mu^{*}, \sigma^{*}\right)$, the information system cannot be carried on.

Result 3: If the Budget Limit $B L$ exceeds the minimal Expected Total Negative Cashflow $B L \geq \mu^{*}$, a risk neutral decision maker will further choose the optimal solution $\left(\mu^{*}, \sigma^{*}\right)$.

\section{Conclusion}

The developed decision model is able to map the existing trade-off between the Expected Losses and the Opportunity Costs of the Regulatory Capital Charge on the one hand as well as the Investments in Security Mechanisms and Insurance Policies on the other hand in a common framework. Thereby, the model optimizes the investments in ex-ante and ex-post controlling mechanisms. Only one $\left(a^{*}, b^{*}\right)$ - respective $\left(S L^{*}, I L^{*}\right)$-combination exists that minimizes the Expected Total Negative Cashflow. Furthermore, the model points out that the constraints - Limits of the Regulatory Capital Charge and Budget Limits - can, under certain conditions, lead to suboptimal solutions. Normally a banking company determines its limits centrally via an individual information system. Not fully taped Limits of Regulatory Capital Charge and Budget Limits cannot be exchanged. Thus, this can lead to inefficiencies from a holistic point of view. The exchange of not fully taped potentials can implicate a greater utility.

However, further research questions arise from the defined assumptions:

- In the model an isolated information system is regarded, by which it is assumed that it is independent of all other systems. Correlations to other information systems are not considered. Taking correlations into account can lead to different results.

- We further assume that Investments in Security Mechanisms and Insurance Policies can be mapped within the domain of $\sigma \in(0, \infty)$. This can be traced back to the property of continuity of the function $\mu(\sigma)$. It is assumed that in reality only discrete action alternatives exist.

- For simplicity, we assumed constant Loss Given Events. However, if the standard deviation of the expected Loss Given Events is taken into account, the Expected Total Negative Cashflow will be affected. A further research topic includes modeling random variables for the loss given events.

\section{References}

[1] Basel Committee on Banking Supervision: Regulatory Treatment of Operational Risk. Working Paper No. 8, 2001.

[2] Basel Committee on Banking Supervision: The New Basel Capital Accord, 2003.

[3] Cruz, A.: Modeling, Measuring and Hedging Operational Risk. John Wiley \& Sons, 2002.

[4] Faisst, U.: Ein Modell zur Steuerung operationeller Risiken in IT-unterstützten Bankprozessen, Multikonferenz Wirtschaftsinformatik, Essen, 2004.

[5] Faisst, U., and Kovacs, M.: Quantifizierung operationeller Risiken - ein Methodenvergleich. Die Bank, Nr. 5, 2003, p. 342-349.

[6] Grzebiela, T.: Insurability of Electronic Commerce Risks. Proceedings of the 35th Hawaii International Conference on System Sciences, 2002.

[7] Karten, W.: Zum Problem der Versicherbarkeit und zur Risikopolitik des Versicherungsnehmers betriebswirtschaftliche Aspekte. Zeitschrift für die gesamte Versicherungswirtschaft, 1979, pp. 279-299.

[8] Müller, G, Eymann, T., and Kreutzer, M.: Telematik- und Kommunikationssysteme in der vernetzten Wirtschaft. Oldenbourg, München, 2003.

[9] Müller, G., and Rannenberg, K.: Multilateral Security in Communications. Vol. 3: Technology, Infrastructure, Economy. Addison-Wesley-Longman, New York, 1999.

[10] Piaz, J.-M.: Operational Risk Management bei Banken. Versus-Verlag, Zürich, 2001.

[11] Rannenberg, K.: Zertifizierung Mehrseitiger Sicherheit: Kriterien und organisatorische Rahmenbedingungen. Vieweg, Braunschweig, Wiesbaden, 1998.

[12] Sackmann, S., and Strüker, J.: 10 Jahre E-Commerce Eine stille Revolution in deutschen Unternehmen. Forthcoming (in german). 